\title{
Spontaneous internal jugular vein thrombosis as primary presentation of antiphospholipid syndrome: case report
}

This article was published in the following Dove Press journal: Vascular Health and Risk Management

\author{
Nabil A Al-Zoubi \\ Department of Surgery, Jordan \\ University of Science and Technology, \\ Irbid, Jordan
}

\begin{abstract}
Introduction: Spontaneous and isolated internal jugular vein (IJV) thrombosis is a rare entity, and atypical localization for venous thromboembolism usually occurs after an oropharyngeal infection, cancer, central venous catheter, and ovarian hyperstimulation syndrome. This report describes a case of spontaneous IJV thrombosis leading to neck pain and swelling as a primary manifestation of antiphospholipid (Hughes) syndrome.

Case presentation: A 44-year-old male with no significant past medical history presented with a 1-week duration of right-sided painful neck swelling. Duplex ultrasound and computed tomography angiogram revealed right IJV thrombosis. Hematology studies were normal apart from positive lupus anticoagulant. The patient was managed conservatively with low molecular weight heparin, antibiotics, and close follow-up.

Conclusion: Acute spontaneous IJV thrombosis as a primary presentation of antiphospholipid syndrome is extremely uncommon. We should be aware of the possibility of this condition in the differential diagnosis and management, as long-term anticoagulation is needed.
\end{abstract}

Keywords: internal jugular vein, antiphospholipid syndrome, lupus anticoagulant

\section{Introduction}

Isolated internal jugular vein (IJV) thrombosis is a rare $^{1}$ but potentially fatal condition due to risk of pulmonary embolism as well as intracranial propagation of the thrombus. ${ }^{2,3}$ It is an atypical localization for venous thromboembolism. ${ }^{4}$ First described in 1912 as a complication of peritonsillar abscess, it is now known to have a varied etiol${ }^{o g y}{ }^{2}$ such as cancer, central venous catheter, and ovarian hyperstimulation syndrome. ${ }^{1}$ The clinical symptoms may be vague and misleading or absent. ${ }^{2}$

Antiphospholipid syndrome (APS), first described in 1983, as thrombosis, abortion, and cerebral disease, is nowadays recognized as a systemic disease with a wide constellation of clinical manifestations related to acute and chronic vascular lesions. ${ }^{5}$

This article presents a case of a 44-year male who presented with right neck swelling and pain caused by a rare entity - IJV thrombosis - and was found to have a positive lupus anticoagulant (LA). To the best of our knowledge, only 3 cases in the literature had been previously reported IJV thrombosis as a primary presentation of APS. ${ }^{6-8}$

\section{Case presentation}

A 44-year-old male, not known to have any medical diseases, was referred to the vascular clinic at King Abdullah University Hospital with right diffuse neck swelling and pain associated with inability to open the jaw properly for the last 7 days. He denied 
any history of infection, trauma, or intravenous drug use. On physical examination, the swelling was tender, diffuse, and without area of fluctuation. He was afebrile with normal vital signs. The patient had neck ultrasound with duplex studies done by the primary care physician which revealed a thrombosed right IJV. Computed tomography angiogram was done, which showed a complete and isolated thrombosis of the right IJV from the base of the skull down to the right subclavian vein (Figure 1). No other pathologies were demonstrated. Ear, nose and throat consultation with direct laryngoscope

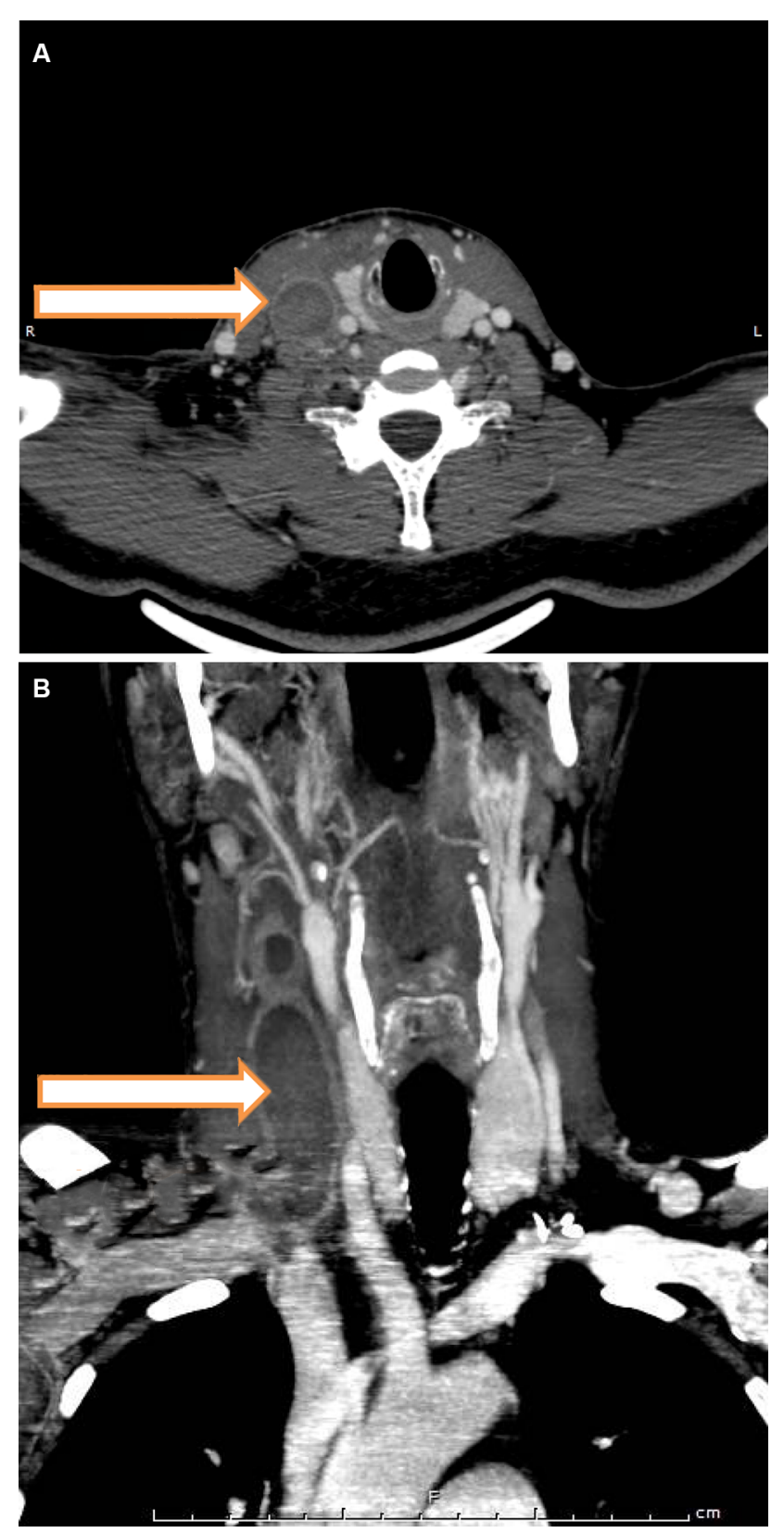

Figure I CTA of the neck revealing thrombosis of the right IJV (arrow); (A) axial and (B) coronal.

Abbreviations: CTA, computed tomography angiogram; IJV, internal jugular vein. revealed no obvious pathology. No pathology was detected in the chest radiography. He was hospitalized and given low molecular weight heparin (LMWH; Enoxaparin sodium $80 \mathrm{mg}$ subcutaneously twice daily) and empirical antibiotics. Routine blood investigations were normal. The thrombophilia profile was normal except for positive LA (activated partial thromboplastin time and tissue thromboplastin inhibition test were used). He had dramatic symptomatic improvement after 6 days and was discharged home on LMWH for 3 months. Then the LA was repeated after 12 weeks, which showed persistent elevation. Then Doppler ultrasound showed organized thrombus in the right IJV. Thus, a decision to treat this patient with long-term oral anticoagulation was taken and he was referred to rheumatology clinic for further follow-up.

\section{Consent}

The patient provided written informed consent for the case report to be published.

\section{Discussion}

Despite an existing anatomical continuity of the venous drainage from the upper half of the body, deep vein thrombosis (DVT) involving IJV, and more proximal veins, seems to bear some different clinical characteristics and affects different groups of patients. ${ }^{4}$ The overall incidence of DVT in the head and neck is under 5\%. ${ }^{9}$ Patients may present with a painful swollen neck or may be asymptomatic. ${ }^{1}$ Suspected IJV thrombosis can be rapidly diagnosed using duplex ultrasonography. Ultrasound has the key advantage of providing a bedside diagnosis with high sensitivity and specificity and may achieve superior resolution to computed tomography in superficial areas. ${ }^{10}$

APS is rare disease (estimated incidence of 5 cases per 100,000 people per year), which affects young adults. ${ }^{5,11}$ It is the most common cause of acquired thrombophilia and accounts for $15 \%-20 \%$ of all episodes of DVT. ${ }^{11}$ It is accompanied by the synthesis of autoantibodies (aPL) against membrane phospholipids and phospholipid-binding proteins which interfere with the coagulation system and increase the risk of arterial thrombosis (stroke, myocardial infarction), venous thrombosis (venous thromboembolic disease), microvascular thrombosis, and obstetrical failures. ${ }^{4}$ Venous thromboses are more common than arterial thromboses in the APS and are reported to involve the deep veins of lower extremities (most common finding), the adrenal, hepatic, mesenteric, portal, or splenic vein, and the inferior vena cava. ${ }^{12}$ APS can occur either as a primary condition or associated with an underlying systemic autoimmune disease, such 
as systemic lupus erythematosus. ${ }^{13} \mathrm{LA}$ is the most specific test for the diagnosis of APS (although transient positivity of antiphospholipid antibodies can be induced by infections or drugs). ${ }^{11}$ APS diagnosis requires at least 1 clinical criterion (thrombotic event or gestational morbidity) and at least 1 laboratory criterion (confirmed positive aPL at 2 or more separate time points with a 12 -week minimum interval) to be fulfilled. ${ }^{11}$ On the other hand, aPL can be detected transiently in some cases, which is clinically insignificant. Therefore, according to the ISTH guidelines, the tests for aPL should be conducted no earlier than 12 weeks following a thrombotic episode, and the positive result should be confirmed after at least 12 weeks. $^{4}$

There are no set guidelines for the treatment for IJV thrombosis, but most reported cases of IJV thrombosis are treated similarly to lower extremity DVTs, primarily with a 3-6-month course of anticoagulation therapy, and in rare selected cases with initial thrombolysis, venous thrombectomy, or placement of a superior vena cava filter when anticoagulation is contraindicated. ${ }^{1}$

In this case, the patient was started on LMWH for 3 months and then shifted to long-term oral anticoagulant when LA was persistently positive. It should be stressed that the presence of LA is associated with high risk of thromboembolic complications. Therefore, continuation of therapy with LMWH during evaluation of APS is acceptable. ${ }^{4}$ After the acute episode, long-term treatment with oral anticoagulant (warfarin with international normalized ratio at or above 3) is the therapy of choice. ${ }^{11,12}$

To the best of my knowledge, only 3 cases in the literature have previously reported IJV thrombosis as a primary presentation of APS. ${ }^{6-8}$

This case illustrates the need for suspecting and recognizing APS early in patients with IJV thrombosis before the diagnosis of idiopathic IJV thrombosis because the treatment and the prognosis are different.

\section{Conclusion}

Acute spontaneous IJV thrombosis as a primary presentation of APS is extremely uncommon. We should be aware of the possibility of this condition in the differential diagnosis and management, as long-term anticoagulation is needed.

\section{Author contributions}

The author contributed toward data analysis, drafting and critically revising the paper and agree to be accountable for all aspects of the work.

\section{Disclosure}

The author reports no conflicts of interest in this work.

\section{References}

1. Javier Corral, Geri Villanueva. A case study of deep vein thrombosis of the right internal jugular vein in a healthy 21 -year-old male. Case Rep Hematol. 2016;2016:3.

2. Shakeel M, Keh SM, Kynaston J, McCluney N, Ah See KW. Evidence based management of spontaneous internal jugular vein thrombosis: a literature review. J Otolaryngol ENT Res. 2015;2(2):00019.

3. Lønnebakken MT, Pedersen OM, Andersen KS, Varhaug JE. Incidental detection of internal jugular vein thrombosis secondary to undiagnosed benign substernal goiter. Case Rep Med. 2010;2010:645193.

4. Mastej K. Recurrent brachiocephalic vein thrombosis in antiphospholipid syndrome. Acta Angiol. 2014;20(1):39-45.

5. Amengual O, Atsumi T. Antiphospholipid syndrome, "the best prophet of the future." Mod Rheumatol. 2018;28(3):409-416.

6. Kale US, Wight RG. Primary presentation of spontaneous jugular vein thrombosis to the otolaryngologist - in three different pathologies. $J$ Laryngol Otol. 1998;112(9):888-890.

7. Tannenbaum LI, April MD, Watts RE, Mary Pearson NM. Thrombosis of the internal jugular vein. Intern Emerg Med. 2016;11(3): 487-488.

8. Tanaka H, Tabuchi K, Nishimura B, Nakayama B, Hara AA. A case of antiphospholipid syndrome with thrombosis of the internal jugular vein as the initial presentation. Pract Otol (Kyoto) Suppl. 2014;140: $120-121$.

9. Shintani T, Matsumoto K, Hattori T, Matsubara K, Watada S, Kitajima M. Spontaneous internal jugular vein thrombophlebitis associated with congenital antithrombin III deficiency. EJVES Extra. 2005;10(2): $51-53$.

10. Chen KH, Chen YJ, Liaw CC, Chang HJ, Yeow KM. Left internal jugular vein thrombosis due to a lung tumor. Chang Gung Med J. 2003;26(6): 458-462.

11. Lopes MRU, Danowski A, Funke A, Rêgo J, Levy R, Andrade DCO. Update on antiphospholipid antibody syndrome. Rev Assoc Med Bras. 2017;63(11):994-999.

12. Hegazi MO, Mourou M, Hassanien OA. Antiphospholipid syndrome presenting with superior vena cava thrombosis. Iran J Immunol. 2009;6(2):103-106.

13. Yang HK, Moon KW, Ji MJ, Han SB, Hwang JM. Primary antiphospholipid syndrome presenting with homonymous quadrantanopsia. Am J Ophthalmol Case Rep. 2018;10:208-210.
Vascular Health and Risk Management

Publish your work in this journal

Vascular Health and Risk Management is an international, peerreviewed journal of therapeutics and risk management, focusing on concise rapid reporting of clinical studies on the processes involved in the maintenance of vascular health; the monitoring, prevention and treatment of vascular disease and its sequelae; and the involvement of

\section{Dovepress}

metabolic disorders, particularly diabetes. This journal is indexed on PubMed Central and MedLine. The manuscript management system is completely online and includes a very quick and fair peer-review system, which is all easy to use. Visit http://www.dovepress.com/ testimonials.php to read real quotes from published authors. 